\title{
ASPECTS DE L'INTERFACE SYNTAXE/SÉMANTIQUE: LE CAS DES VERBES À /COMPLEXITÉ/
}

\begin{abstract}
A bstract. Bazenga Aline, Aspects de l'interface syntaxe/sémantique: le cas des verbes à /complexitél [Aspects of the interface syntax/semantics: a case of the complexity verbs]. Studia Romanica Posnaniensia, Adam Mickiewicz University Press, Poznań, vol. XXXI: 2004, pp. 155-165. ISBN 83232-1353-4, ISSN 0137-2475.
\end{abstract}

The /complexity/ feature (Blanche-Benveniste et al. 1987) incorporates a class of French verbs known as 'symmetric' verbs (rivaliser, cohabiter), 'reciprocal' verbs ( $s$ 'entraider) without, however, being restricted solely to those classes (cf. grouiller, amonceler, succéder, grouper, etc.). This feature induces a 'plural' reading of the entity formed by the verbal lexeme and the syntactic positions it creates. In this paper, we intend to put forward a new descriptive format for such verbs, using the notion of 'verbs operators' (cf. Bach et al. 1995) and based on a combination of lexical, syntactic and semantic criteria (derivational affixes, Prep-Constructions, Se-Constructions, Coordination).

\section{INTRODUCTION}

Le trait /complexité/ permet de réunir des verbes que l'on retrouve distribués sous différentes typologies verbales existantes portant, soit sur les types de procès (états vs activités, etc.) soit sur des structures syntaxiques et actancielles (verbes monovalents, divalents, trivalents) soit encore sur des ensembles lexicaux (verbes d'existence, verbes psychologiques, verbes de mouvement, etc.). Cette capacité à intégrer différentes typologies est due, me semble-t-il, à leur l'hétérogénéité tant sur le plan lexical que sur le plan syntaxique. Il me semble que le recours aux critères sur lesquels s'appuient ces différentes typologies ne permet de traiter que des aspects ponctuels, concernant tel ou tel sous-ensemble de verbes. L'objectif de cette communication est de présenter, sous forme d'hypothèses descriptives, un ensemble d'outils analytiques capable de rendre compte de la multiplicité des configurations se rapportant à /complexité/ et de la ramener à un ensemble réduit de principes. 


\section{LES VERBES A.COMPLEXITÉ/}

\section{Verbes à /complexité/ (Blanche-Benveniste et alii, 1987)}

i) Sélection d'un élément de valence (sujet ou complément) obligatoirement pluriel (ou collectif ou constitué d'éléments coordonnés);

ii) En absence de cet élément de valence au pluriel, par la présence d'un complément prépositionnel en relation de solidarité avec un élément de valence, sujet ou complément, au singulier et l'interprétation nécessaire d'un singulier comme ayant une valeur de 'collectif'.

Tableau 1

\begin{tabular}{|c|c|c|c|c|c|c|c|c|}
\hline \multirow{3}{*}{ Verbes } & \multirow{3}{*}{ Formulations } & \multicolumn{7}{|c|}{ PLACES DE VALENCE } \\
\hline & & \multicolumn{2}{|c|}{$\mathbf{P} \boldsymbol{\theta}$} & \multicolumn{2}{|c|}{ P1 } & \multirow{2}{*}{$\begin{array}{c}\text { P2 } \\
\text { LUI }\end{array}$} & \multirow{2}{*}{$\begin{array}{c}\text { P3 } \\
\text { DE LUI }\end{array}$} & \multirow[t]{2}{*}{ AVEC + } \\
\hline & & $\begin{array}{c}\text { sing. } \\
\text { IL }\end{array}$ & $\begin{array}{c}\text { plur. } \\
\text { IISS }\end{array}$ & $\begin{array}{l}\text { sing. } \\
\text { LE }\end{array}$ & $\begin{array}{l}\text { plur. } \\
\text { LES }\end{array}$ & & & \\
\hline converger & $\mathbf{I}$ & & ++ & & & & & \\
\hline alterner & $\begin{array}{l}\text { I } \\
\text { II }\end{array}$ & + & + & & & & & $+\ldots$ \\
\hline entr'aider & I & & + $-\mathbf{S E}$ & & & & & \\
\hline quereller & $\begin{array}{l}\text { I } \\
\text { II }\end{array}$ & $+-\mathrm{SE}$ & 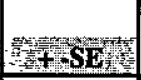 & & & & & $4+$ \\
\hline rencontrer & I & + & 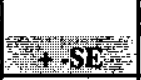 & $\operatorname{mot}+$ & & & & \\
\hline grouper & $\begin{array}{l}\text { I } \\
\text { II } \\
\text { III } \\
\end{array}$ & $\begin{array}{l}+ \\
+\end{array}$ & H & + & +4 & & & $4+4$ \\
\hline ressembler & $\begin{array}{l}\text { I } \\
\text { II }\end{array}$ & + & $Q+$ SE & & & + & & \\
\hline séparer & $\begin{array}{l}\text { I } \\
\text { II } \\
\text { III }\end{array}$ & $\begin{array}{l}+ \\
+\end{array}$ & ${ }^{n}+\mathbf{S E} \mathbf{E}^{-2}$ & + & + & & $=+4$ & \\
\hline
\end{tabular}

Verbes à /complexité/

Ces propriétés permettent d'intégrer les verbes tels que:

- propriétés i) et ii)

(1) a. Ils voisinent (ensemble)

b. Il voisine avec elle

(2) a. Ils diffèrent

b. Il diffère de lui

(3) a. Ils se ressemblent

b. Il ressemble à celui-là 
(4) a. Je les groupe

b. Je le groupe avec ça

(5) a. Je les sépare

b. Je le sépare de celui-là

La propriété i) est illustrée par a [cf. (1) - (5)], la propriété ii) par b [cf. (1)(5)]. Entre les propriétés i) et ii) il doit être établi une hiérarchie, i) étant prépondérante par rapport à ii). Certains verbes ne satisfont que la propriété i):

\section{- propriétés i)}

(6) a. Ils s'entraident

b. * Il s'entraide avec elle

(7) a. Ils grouillent/fourmillent dans le jardin

b.* Il grouille avec celui-là dans le jardin

cf. abonder, fourmiller, éparpiller, collectionner, disseminer, atrouper, etc.

Et cela constitue une condition suffisante d'intégration de la classe des verbes à /complexité/; D'autres verbes en sont exclus:

(8) a. Je les regarde

b. je le regarde

(9) a. Ils sautillent dans le jardin

b. Elle sautille dans le jardin

\section{HYPOTHESE: CONFIGURATIONS SYNTAXIQUES ET POINTS D'ANCRAGE DE /COMPLEXITÉ/}

- 'syntaxe de position' (Skarup 1975; Willems 1993)

Je distingue dans la syntaxe verbale deux zones syntaxiques - Sujet et Objet ${ }^{1}-$ organisées autour du noyau verbal. La zone Objet, contrairement à la zone Sujet, peut comprendre plus d'une position syntaxique. Le trait de /complexité/ se réalise soit en Sujet:

\section{Position Sujet}

(10) a. ces deux artistes collaborent

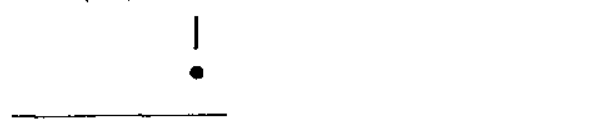

' Cette notion s'inspire du concept de 'syntaxe de position' (Skarup, 1975). Cf. également Willems (1993). 
b. les étudiants s'entraident

I

Soit dans la zone Objet et dans différentes positions syntaxiques:

\section{Position Objet}

(11) a. Je groupe les figures

b. le prisme décompose la lumière en radiations simples

c. Cette feuille grouille $d$ 'insectes

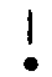

Le trait /complexité/ peut également s'ancrer sur deux positions:

\section{Positions Sujet et Objet}

(12) a. Ceci diffère de cela

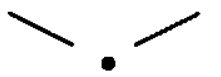

b. Je combine ceci avec cela

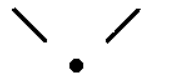

\section{HYPOTHÈSE: 'OPERATEURS VERBAUX' DU TRAIT [+DISCRET]}

- A-quantification (Bach et al. 1995); 'opérateurs verbaux' (Partee 1995)

$\Rightarrow$ 'opérateurs de complexité': [+discret]

- Relative elaboration of events (Kemmer 1994)

$\Rightarrow$ échelle de gradation de [+discret]

L'analyse des verbes à /complexitél doit être menée dans le cadre d'une sémantique du 'non-singulier' (Dougherty 1970) ou d'une sémantique du 'plus d'un'. Selon Bach et al. (1995) l'expression de la quantification se fait à partir de deux catégories: la quantification $\mathrm{D}$ et la quantification $\mathrm{A}$ ( $D$-quantification et A-quantification). La quantification-D s'applique au domaine des SN, i.e. par les constituants de la classe des déterminants et des noms; La quantification-A s'exprime au niveau de la phrase par des adverbes (toujours, normalement, etc.) les quantificateurs 'flottants' (chaque), les auxiliaires, les affixes et autres arguments- 
structure adjusters. Tandis que la première catégorie est constituée d'une classe fermée, la seconde est ouverte et hétérogène.

Je propose une description formelle inspirée du concept de A-quantification et qui inclut la notion de 'opérateurs verbaux' (Partee, 1995: 559). Dans le cas des verbes à /complexité/, ces opérateurs seront désignés par 'opérateurs de complexité'. Les 'opérateurs de complexité' correspondent donc à des éléments formels qui, aussi bien au niveau de la structuration lexicale des items verbaux qu'au niveau de leurs propriétés syntaxiques, contribuent à l'interprétation 'plus d'un'. Je traduis cette propriété par un trait constitutif de /complexité/: le trait [+discret]. Ce trait dénote non seulement la valeur 'plus d'un' mais également la notion de délimitation, délimitation externe - ou bornes qui configurent l'extension de /complexité/ - et délimitation interne, i.e. les limites entre les unités faisant partie de /complexité/.

\section{Cf. Bunt (1985).}

Hypothèse de la référence homogène:

'Mass-nouns refer to entities as having a part-whole structure without singling out any particular parts and whithout making any commitments concerning the existence of minimal parts' (Bunt: 1985: 46) mais aussi Landman approche.

Cf. Chierchia (1995: 2).

Tous les noms ont des parties minimales, même les noms massifs comme water (de l'eau)

- 'inherent plurality hypothesis'

'A mass noun simply denotes a set of ordinary individuals, plus all the pluralities of such individuals. For example 'change' denotes, roughly, single coins and the possible sets of pluralities of coins. This view is an 'atomistic' one: we are committed to claim that for each mass noun there are minimal objects of that kind, just like for count nouns, even if the size of these minimal parts may be vague'

Cf. Link (1983) défend également ce point de vue, Krifka (1992).

Je propose également d'associer la notion de 'relative elaboration of events' proposée par Kemmer (1994) dans le cadre de l'analyse de la voix moyenne, au trait [+discret], en l'insérant dans une échelle de gradation. Cette option part du présupposé que la valeur de 'plus d'un' inhérente au trait /complexité/ est de nature discrète, et que les différences observables dans différents types de contextes verbaux peuvent être calculées en termes de degrés de [+discret].

Cf. également concept théorique d'échelle Horn (1972), Ducrot (1973) Ancombre et Ducrot (1978), Fauconnier (1976, 1980). 
L'échelle reflète une certaine hiérarchie entre les prédicats; elle est une relation d'ordre: chaque élément posé sur l'échelle marque le degré d'intensité quantitative.

Fauconnier (1976) considère une échelle comme 'un ensemble ordonné $\{\mathrm{m}<$ $\ldots<\mathrm{x} 1<\mathrm{x} 2<\ldots<\mathrm{xn}\}$ dont on se sert surtout pour les catégories orientées, comme par exemple:

1) adjectifs, p. ex.: intéressant et passonnant (passionnant $>$ intéressant)

2) les adjectifs accompagnés de certains adverbes qui marquent l'orientation, comme p. ex. les adverbes presque, très, plus

Enfin, les 'opérateurs de complexité', ou les différents ajusteurs du trait [+discret] considérés, se situent à différents niveaux: au niveau de la composition morphologique des items lexicaux, au niveau de la sélection des constructions syntaxiques des items verbaux (cf. constructions prépositionnelles, constructions en Se) et au niveau des configurations nominales des positions syntaxiques de chaque construction verbale (i.e. les positions Sujet et Objet).

\subsection{AFFIXES DERIVATIONNELS}

Dans la configuration morphologique d'une unité lexicale, telle que [préf-Xsuff], nous pouvons y délimiter trois points d'ancrage du trait [+discret]. Celui-ci peut être interprété par un élément préfixal, comme par exemple en (13a), par le préfixe Com-, réalisé Co- ou en (13b) par le préfixe Entre- :

(13) a. Cohabiter

b. entrelacer

Dans les deux cas, les préfixes dénotent une valeur plus abstraite - [+discret] et une valeur propre: 'comitative' pour (13a) et 'réciproque' pour (13b).

Le trait [+discret] peut encore être identifié dans les bases nominales renvoyant à la notion de 'multiplicité':

(14) a. fragmenter; segmenter

b. collectionner; peupler

Cette 'multiplicité' étant perçue du point de vue des 'parties de' (cf. fragment, segment, en (14a)) ou du point de vue de l'ensemble', ou du 'tout' (cf. collection, peuple, en (14b)).

\subsection{CONSTRUCTIONS PRÉPOSITIONNELLES}

Les verbes à /complexité/ comportent un nombre important de verbes qui sélectionnent des constructions prépositionnelles. 
Les prépositions, en tant qu'éléments 'opérateurs de complexité' partagent avec les autres opérateurs (par exemple, les préfixes, cf. 3.1) le fait d'être porteurs de deux valeurs: une valeur abstraite $-[+$ discret $]-$ et une valeur liée au contexte de réalisation. La valeur de [+discret] se traduit par la capacité de ces éléments formels à introduire les limites externes des structures syntaxiques où elles peuvent figurer. Le calcul de la signification de chaque préposition est effectué en ramenant les différentes significations contextuelles à une signification prototypique ${ }^{2}$.

Les groupes prépositionnels peuvent constituer à eux seuls le noyau de réalisation de /complexité/ [cf. exemple (11b)] ou s'associer à d'autres positions syntaxiques dans une structure à ramification binaire [cf. exemples (12a et 12b)].

Associées à ces positions syntaxiques, parmi les verbes à /complexité/ qui ont la possibilité de sélectionner une construction prépositionnelle, il faut encore considérer deux types: les verbes qui ne sélectionnent qu'une construction prépositionnelle, à préposition fixe (rivaliser avec) et les verbes qui sont susceptibles de se combiner avec plusieurs constructions prépositionnelles (lutter avec/contre):

(15) a. Rivaliser [ $\varnothing$, avec [ Sujet]]: Pierre rivalise avec Jean b. Lutter $[\varnothing$, avec, contre [ Sujet]]: Pierre lutte avec /contre Jean
[+prép, +prép]
$[\varnothing,+$ prép $]$
$(+)$

Par cette représentation je veux rendre compte de l'absence d'alternance de préposition significative (15a) et l'alternance de préposition, elle aussi significative, en (15b).

Pour les verbes qui ne sélectionnent pas de constructions prépositionnelles, le trait [+discret] est assuré au niveau de la syntaxe par un autre opérateur, le clitique Se, celui-ci étant marqué, tout comme la préposition, par deux types de valeur: une valeur générale, celle de délimitation et des valeurs propres, calculées en fonctions des contextuelles (Se-réciproque, Se-passif, etc.).

Constructions en Se [+discret]

Deux participants

Un participant

(Initiator/Endpoint)

(Initiator)

Const. Transitive Se réciproque Se-réflechi Se-moyen Se-ergatif Const. Intransitive

degré de élaboration des événements

${ }^{2}$ Pour les notions de sens abstrait, sens prototypique et sens contextuels des prépositions, cf. Englebert (1993). 


\subsection{CATÉGORIES NOMINALES DES POINTS D'ANCRAGE DE/COMPLEXITE/}

Les catégories nominales que j'ai sélectionnées - SNpluriel, Ncollectif, SN complexe en $\mathrm{Et}$ - partent de l'observation de leur distribution dans les contextes verbaux marqués par/complexité/. Ainsi, par exemple:

(16) a. Pierre et Jean rivalisent

b. Les deux frères rivalisent

c. ? La foule rivalise

Les verbes 'symétriques', tel que rivaliser ainsi comme les verbes à lecture réciproque semblent être incompatibles avec des positions syntaxiques occupées par des noms collectifs (16c). D'autres verbes, tels que grouiller (mais aussi, et entre autres, disperser, éparpiller):

(17) a. ${ }^{*}$ La fourmi et la grenouille grouillent dans le jardin

b. Les fourmis et les grenouilles grouillent dans le jardin

c. Les fourmis grouillent dans le jardin

d. La foule grouillait sur la place

acceptent bien des nominaux de type collectif (17d) mais imposent des restrictions en ce qui concerne SN complexes en ET (cf. (17a) et (17b)).

Enfin, les verbes tels que collectionner:

(18) a. Je collectionne des timbres

b. Je collectionne le timbre et la monnaie

c. Je collectionne *le mobilier/? du mobilier

semblent difficilement compatibles avec des positions syntaxiques de type collectif (18c) tout en acceptant bien les autres catégories nominales.

A ce niveau, et comme pour les autres opérateurs de [+discret], j'établit une échelle qui va du [+discret] au [-discret], comme ci-dessous:

Tableau 2

[+discret]

[-discret]

$[+$ NomPropre, + Et $] \quad[+\mathrm{SN} s i n g,+\mathrm{Et}] \quad[+\mathrm{SNpl},+\mathrm{Et}] \quad[+\mathrm{Ncoll},-\mathrm{Et}]$ 


\section{HYPOTHĖSE: PRINCIPE ORGANISATEUR DE [+DISCRET] OU SON ARTICULATION AVEC LA SÉMANTIQUE DES RELATIONS D'INCLUSION}

- taxonomies sémantiques des relations d'inclusion (Winston et al. 1987). $\Rightarrow$ articulation [+discret] - [+inclusion]

Les données obtenues au niveau de l'analyse des 'opérateurs verbaux' de [+discret] des verbes à /complexitél sont intégrées, au niveau sémantique, dans le cadre des taxonomies sémantiques des relations d'inclusion telles qu'elles sont définies par Winston et al. (1987). Les auteurs distinguent sous cette catégorie trois sous-types de relations d'inclusion: inclusion de classe, inclusion spatiale et inclusion méronymique. Les relations méronymiques ou 'relations partie-de' sont définies par trois propriétés essentielles: transitivité, non-reflexivité et antisymétrie. Ce type de relations sémantiques est extrêmement général et qui peut s'appliquer notamment au domaine matériel et temporel et me semble assez fonctionnel et capable d'absorber les données concernant/complexité/. Ainsi, si par les échelles de [+discret] on obtient les 'grains' ou la 'texture' de /complexitél, à travers l'application du paramètre 'relation d'inclusion', on peut arriver à la détermination d'un certain nombre de propriétés concernant son organisation interne. Pour déterminer les différents types de relations sémantiques, je fais appel de nouveau, aux propriétés sémantiques des éléments formels sélectionnés comme opérateurs de [+discret] (affixes, prépositions, catégories nominales). À titre d'exemple, /complexité/ peut se traduire au niveau de sa structure interne par:

(19) a. rimer [+méronymique, + Sujet, associatif (avec)]

b. différer [+méronymique, + Sujet, dissociatif(de)]

c. lutter[+méronymique, + Sujet, associatif (avec), oppositif (contre)].

Les trois verbes de (19) ont une organisation interne de type méronymique, i.e. 'relation partie de', cette relation pouvant être caractérisée par une relation 'd'association'en (19a), de 'dissociation' en (19b) ou 'd'association et opposition 'en (19c).

\subsection{LA NOTION DE 'VUES DE PLURALITÉS’}

\section{- notion d'event views (Crof 1990)}

La sémantique proposée pour les verbes à /complexité/ se définit par rapport au paramètre appelé relations sémantiques d'inclusion. Cela veut dire qu'elle peut être définie soit négativement, en l'absence de relations sémantiques d'inclusion, soit positivement, par la sélection d'un des types de relations sémantiques d'inclusion. 
Un verbe marqué par /complexité/ peut développer plusieurs types de relations du [+discret], chaque type constituant une 'vue' sur/complexité/, ou mode d'organisation interne de 'plus d'un'. J'utilise le terme 'vue' dans le sens qui lui est attribué par Croft (1990: 263) dans sa théorie de la Structure Causal des Evénements, et où les constructions causatives, inchoatives et résultatives constituent the three VIEWS of a simple event, or EVENT VIEWS. Ainsi, un verbe tel que verbe grouiller:

(20) a. une vue [-méronymique, +cumulatif $\left({ }^{*}\right.$ SNsg ET)]: Les fourmis/ *la fourmi et la grenouille grouillent dans le jardin

b. une vue [-méronymique, +inclusion topologique(de)]: Le jardin grouille de fourmis

est caractérisé par l'ensemble des 'vues de pluralités' constitué par les deux vues (20a et 20b), toutes les deux non-méronymiques, (22a) contribuant avec sa vue de type 'cumulatif' et (22b) avec sa vue 'inclusion topologique'.

\section{CONCLUSIONS}

En cette première approche de l'analyse des verbes à /complexité/j'ai postulé quelques hypothèses descriptives dont l'objectif est de déterminer le principe organisateur du sens de /complexité/. Ces hypothèses essaient d'établir un lien entre la syntaxe et la sémantique verbale par l'articulation de deux traits constitutifs: [+discret] et [inclusion] le premier étant soumis à une échelle de gradation, le deuxième, servant à définir les types de relations entre les unités, étant soumis aux conditions de sélection/non-sélection.

Cette approche s'inspire, par le caractère binaire des outils descriptifs et par l'articulation des différents niveaux d'analyse, de celle formulée par Jackendoff (1990), dans le cadre de la sémantique conceptuelle, où les deux domaines nominal et verbal - sont reliés autour de la notion d'entités conceptuelles' et par un système de traits utilisant la notion de limite [ \pm boundeness] et de structure interne [ tinternal structure].

\section{RÉFÉRENCES BIBLIOGRAPHIQUES}

Bach, E., Jelinek, E., Kratzer, A. et Partee, B. (éds) (1995), Quantification in Natural languages, vol. I. Dordrecht-Boston-London: Kluwer Academic Publishers.

Blanche-Benveniste, Cl. Deulofeu, J., Stéfanini J. et van Den Eynde, K. (1984), Pronom et syntaxe, l'approche pronominale et son application en français. Paris: SELAF.

Croft, W., 1996 (1990), Typology and Universals. Cambridge: Cambridge University Press. 
Dougherty, R. C. (1970), A Grammar of Coordinate conjoined Structures: 1 , Language, vol. 46, 4: 850-898.

Englebert, A. (1992), Le petit mot DE: étude de sénnatique diachronique. Genève-Paris: Droz.

Jackendoff, R., (1991), Parts and Boundaries, Cognition, 41: 9-45.

Ke mmer, S. (1994), Middle voice, transitivity and the elaboration of events, B. Fox et P. J. Hopper (éds), Voice: form and function. Amsterdam-Philadelphia: John Benjamins, 179-230.

Skarup, P. (1975), Les premières zones de la proposition en ancien français. Essai de syntaxe de position, Revue romane, $6, \mathrm{n}^{\circ}$ spécial (Coll. Études romanes).

Willems, D. (1993), L'impact de l'ordre des mots sur la prédication. La relation sujet-prédicat, Travaux de Linguistique, 26: 89-101.

Winston, M. E., Chaffin, R. et Herrmann, D. (1987), A Taxonomy of Part-Whole Relations, Cognitive Science, 11: 417-444. 